\title{
SOLID STATE SENSOR FOR LOCATING AND IMAGING SOURCES OF GAMMA AND X-RADIATION
}

\author{
S. Kronenberg, G.J.Brucker, and E. Bechtel \\ US Army Project Manager NBC Defense systems, AMSCB-PM-NNN-F, \\ Fort Monmouth, N.J.
}

\begin{abstract}
This paper describes the design and characterization of a directional Solid state Detector (SSD) that generates images of radiation point sources and scatter patterns from irradiated targets, thus accurately identifying their locations. Previous papers demonstrated that other types of directional radiation sensors, such as Ionization Chambers, GeigerMueller and Scintillation Counters, can be designed to detect and locate arrays of gamma ray and $x$-ray point sources and broad scatter patterns.
\end{abstract}

\section{Introduction}

The initial work $(1,2)$ in the development of directional detectors of gamma and $x$-ray sources involved three types of directional sensors: Ionization Chamber (IC), GeigerMueller counter (GM), and Scintillation Counter (SC). They utilized planar high/low atomic number materials on either side of the detector type. Experiments conducted with those sensors demonstrated that they have $4 \pi$ solid angles of acceptance and that arrays of discrete point sources of cesium, cobalt, and $x$-rays can be detected and located accurately by angle and distance. The angular locations of the sources were determined from either the scan data or the first derivative of these data. The intensities of the sources were shown by an analytical derivation (2) to be proportional to the first derivatives of the scan data.

In those investigations, we demonstrated that an angular resolution of 1.25 seconds of arc was achievable (1) with the IC sensor. The SC detector (a scintillator package consisting of 12 sandwiches of lucite/ scintillator/lead layers on a two inch photomultiplier tube) was shown to be capable of locating a cesium source with a dose rate of 13 $\mu \mathrm{rads} / \mathrm{h}$ at the detector location (2). That sensor's sensitivity was increased by using a much larger scintillator and photomultiplier tube. The sensitivity of that larger device (3) was shown to be 6 counts/photon- $\mathrm{cm}^{2}$ (thus capable of locating a cesium source with a dose rate of 3 urads $/ \mathrm{h}$ ). That work was expanded in this study to demonstrate that a Solid state Detector (SSD) sandwiched between layers of high/low $z$ materials can be designed to generate images of radiation point sources and broad patterns of scattered photons. Experiments showed that the SSD sensor was able to see through walls of concrete and to locate point sources in the same manner as previous designs (2). The objective of this report is to present the results of this latest research.

\section{Principle of Directional Photon Sensing}

The principle of operation and design of directional sensors used in this study was described in reference (1) and (2); however, for the convenience of this paper's readers a part of that text is repeated here. A planar detector (e.g. a layer of scintillator) which is thin compared with the applicable ranges of the photo-Compton electrons is sandwiched between plates of low and high $\mathrm{Z}$ (atomic number) materials. For quantum energies of gamma rays greater than $0.6 \mathrm{MeV}$, the number of electrons emitted from the surfaces of the two layers into the detector is greater when the gammas enter through the low $\mathrm{z}$-layer than when they enter through the high $\mathrm{z}$-layer (2). For lower quantum energies the emission effect is reversed, but now the incident gamma rays are attenuated more by the high z-layer than the low $z$-layer. Thus the signal is again greater when the gamma rays enter through the low $\mathrm{z}$-materials compared to the signal when the gamma 
rays enter through the high z-layer. The worst case energy is about 0.6 $\mathrm{MeV}$ which is close to the 0.662 gamma rays emitted by ${ }^{137} \mathrm{Cs}$. Thus, if the sensor works for a cesium source then it will work for a majority of other quantum energies.

\section{Experimental Description}

The detector part of the SSD is a Canberra PIPS (B300AM) device, $385 \mathrm{~km}$ thick, fully depleted $\mathrm{n} / \mathrm{p}$ diode with an active area of $300 \mathrm{~mm}^{2}$ that is designed for counting beta particles. To use it as a directional detector, the standard design was modified by adding lead and tissue equivalent plastic (high/low $z$ combination) to either side of the detector. Preliminary experiments were made with this modified design (hereafter referred to as "old SSD"). The silicon wafer was then removed from its housing and mounted in an enclosure which consisted of $2.51 \mathrm{~mm}$ thick wall of lead on one side and of $2.31 \mathrm{~mm}$ thick wall of plastic on the opposite side. Conductive rubber orings that were removed from the original housing made electrical
Concrete blocks were placed between a ${ }^{60} \mathrm{Co}$ source and the SSD. The sensor scanned the incident gamma rays as it rotated through $360^{\circ}$. The Scintiliation counter (SC)

directional detector (previously used and described in reference 2) was also used to scan the cobalt source in the same experimental setup. The results of this new SSD design were then compared to the standard SC detector which served as our reference.

Sources of ${ }^{137} \mathrm{Cs}$ and low energy $\mathrm{x}-$ rays were also scanned by the old and new SSDs. The $x$-ray source is an industrial $300 \mathrm{KV}$ machine made by Sieffert Inc of Germany. The derivatives (in this experiment) were determined by curve fitting second order regression functions to appropriate regions of the scan response. Although the angular position of the source can be determined accurately by either the direct scan data or the derivative data, the most accurate design was demonstrated by the null method described and used in applications of the directional ionization chamber (1).

1- Si water

2- Si active region

3- Conductive rubber rings

4- $\mathrm{Pb}$ housing

5- Plastic housing

6- Air space

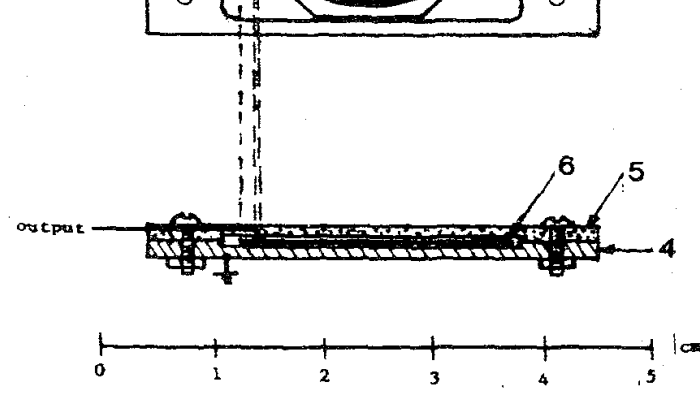

FIG. 1 SOLID STATE DIRECTIONAL DETECTOR

connections to both sides of the $\mathrm{Si}$ wafer in its active region as is shown in Fig. 1. The thickness of the rubber rings resulted in an air gap of $495 \mathrm{~mm}$ on each side. This repackaged version is referred here to as the "now SSD".

\section{Results of Experiments}

Figure 2 shows the plot of the SC scan data as well as the derivative of these data for the concrete block setup. The corresponding results obtained with the SSD sensor are 
shown in Fig. 3. Figure 4 displays the scan data of $174 \mathrm{keV} x$-rays taken with the "old SSD". The scan results of a ${ }^{137} \mathrm{Cs}$ point source that were obtained by the old and new SSDs are compared in Fig. 5. A comparison of new SSD scans for a low and a high intensity point source of ${ }^{137} \mathrm{Cs}$ is shown in Fig. 6 .

\section{Discussion}

The SC scan measurements in Fig. 2 show that at $135^{\circ}$ a change in the response slope points out the location of the edge of the concrete block and that the scattered radiation is now entering parallel to the lead emitter of the detector. The slope remains constant for $45^{\circ}$ until $180^{\circ}$ is reached, then a sharp change in slope occurs. This new slope represents the region of the unscattered radiation, starting at an angle of $180.25^{\circ}$. When the detector reaches $180.5^{\circ}$, the change of slope is towards a shallower decrease. This shallower decrease is in the region of the scatter pattern from the remainder of the block, following the pattern of unscattered radiation. In the experimental setup, the primary source was lined up with the center of the blocks and the angle subtended at the detector was $90^{\circ}$, that is $45^{\circ}$ to either side of the primary beam. The derivative of the scan clearly shows the occurrence of the transition regions at the edges of the concrete and the primary beam.

The subsequent rotation of the sensor causes the response to level out as the detector is exposed to a constant mixture of scatter (this also includes the scatter from the walls and other objects in the room) and primary radiation. As the rear. edge of the detector comes around, the mirror image begins to take shape at about $315^{\circ}$ where the forward scatter from the concrete generates a transition slope in the range of $315^{\circ}$ to $360^{\circ}$. This slope terminates at $360^{\circ}$ and is then followed by the change in slope due to the primary radiation region $\left(360.25^{\circ}\right.$ to $\left.360.5^{\circ}\right)$. It can be seen from both of the derivative plots (i.e. direct and mirror image derivatives) that the location of the source is at $180^{\circ}$.

Figure 3 containg a plot of the scan results for the same setup as in the SC experiment that were taken with the old SSD sensor. The location of the point source is clearly indicated in the response curve similar to the SC sensor. The poorer angular resolution of the SSD sensor in locating the concrete-scatterpatterns and the primary photons compared to the $\mathrm{SC}$, is very apparent from Figs. 2 and 3 . However, the primary source was still located by the SSD to within about two degrees of its location behind the concrete blocks. In the case of the determination of the blocks angular width from the scan data, the accuracy was within about $5^{\circ}$ as indicated in Fig. 3.

The gamma rays from cobalt have an average energy of $1.25 \mathrm{MeV}$. To demonstrate the directional property for lower quantum energies, the old SSD was used to scan various effective $x$-ray energies, ranging from 14 to $250 \mathrm{keV}$. Figure 4 shows the data that were obtained by the SSD in scanning the $x$-ray source of $174 \mathrm{keV}$ (effective energy obtained with specific, calibrated filters). The characteristic sharp transitions at $90^{\circ}$ and $270^{\circ}$ (direct and mirror images) indicate the location of the focal spot on the x-ray tube's tungsten target. Similar detector responses were obtained for the other $\mathrm{x}$-ray energies as well.

The shape of the response curve was improved by removing the silicon detector from its steel container and repackaging it between lead and tissue equivalent plastic as shown in Fig. 1. This container design allowed the lead and plastic layers to be close to both sides of the silicon wafer without the intervention of the steel wall. The scan data of a ${ }^{137} \mathrm{Cs}$ point source that were taken by the old and new SSDs are compared in Fig. 5. The new SSD has a flatter scan curve in the regions between the transitions at $0^{\circ}$ and $180^{\circ}$ (source locations) compared to the response of the old SSD. In addition, the angular resolution of the new SSD is better than the old one. The removal of the original steel container was probably responsible for the change in the shape of the scan response.

Figure 6 shows scans of a cesium source by the new SSD for a low and a high dose rate of $2 \mathrm{mrads} / \mathrm{hr}$ and 1 rad/h, respectively. The data were obtained by simply scanning the same source with the detector located at two different distances from the source. It can be seen from Fig. 6 that the angular resolution for the 


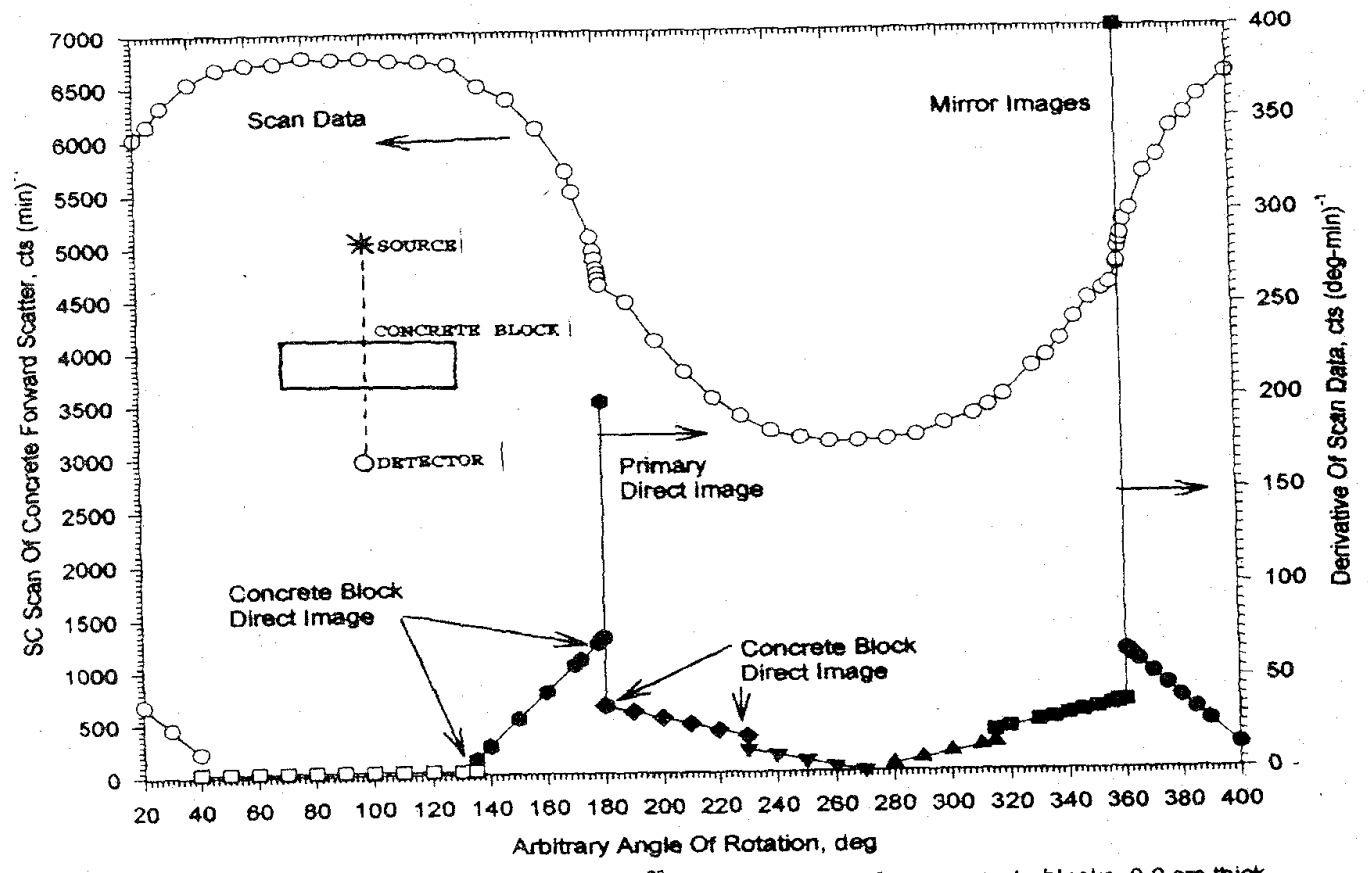

Fig. 2 Scintillation Counter (SC) Bcan of forward ${ }^{\infty} \mathrm{Co}$ photon scatter from concrete blocks, $9.2 \mathrm{~cm}$ thick.

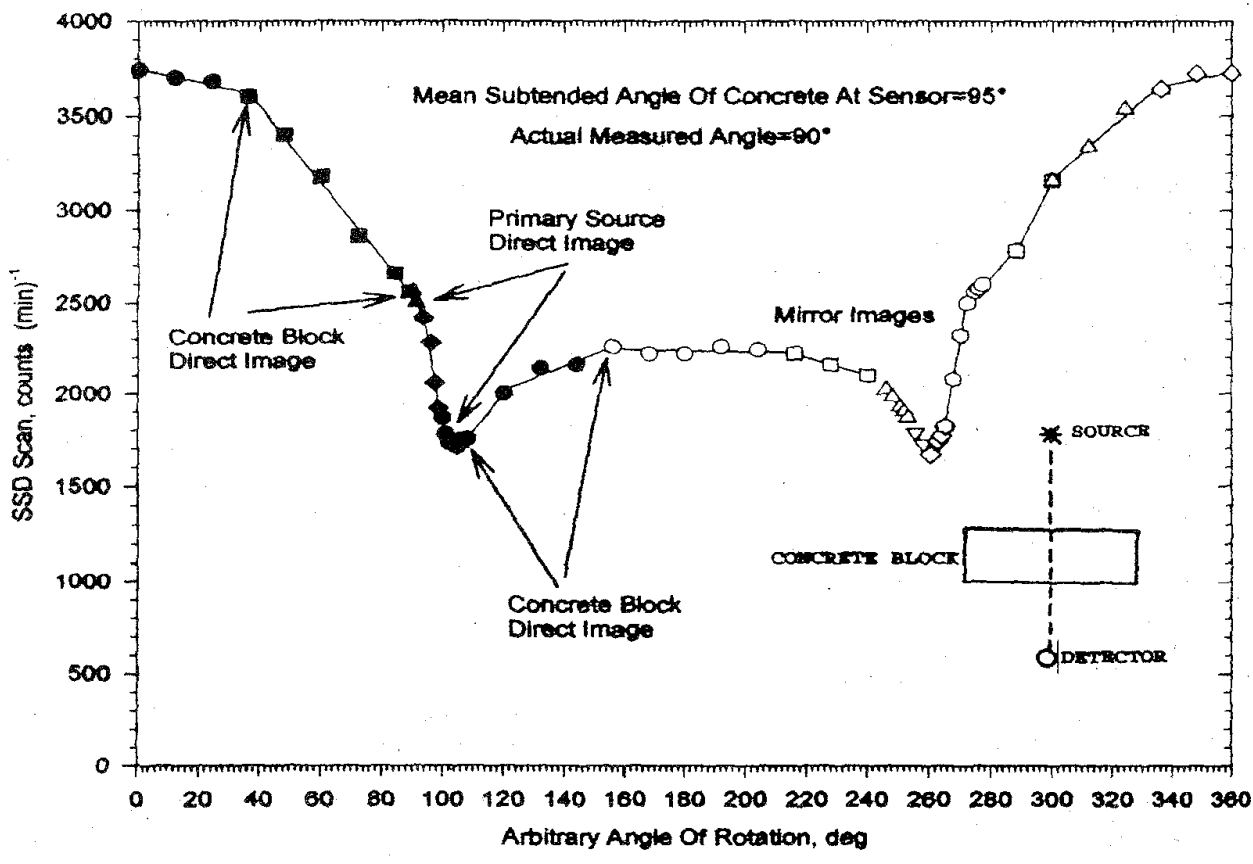

Fig. 3 Solld state Detector scan of forward ${ }^{\infty} \mathrm{Co}$ photon scatter from concrete blocks, $9.2 \mathrm{~cm}$ thick. 


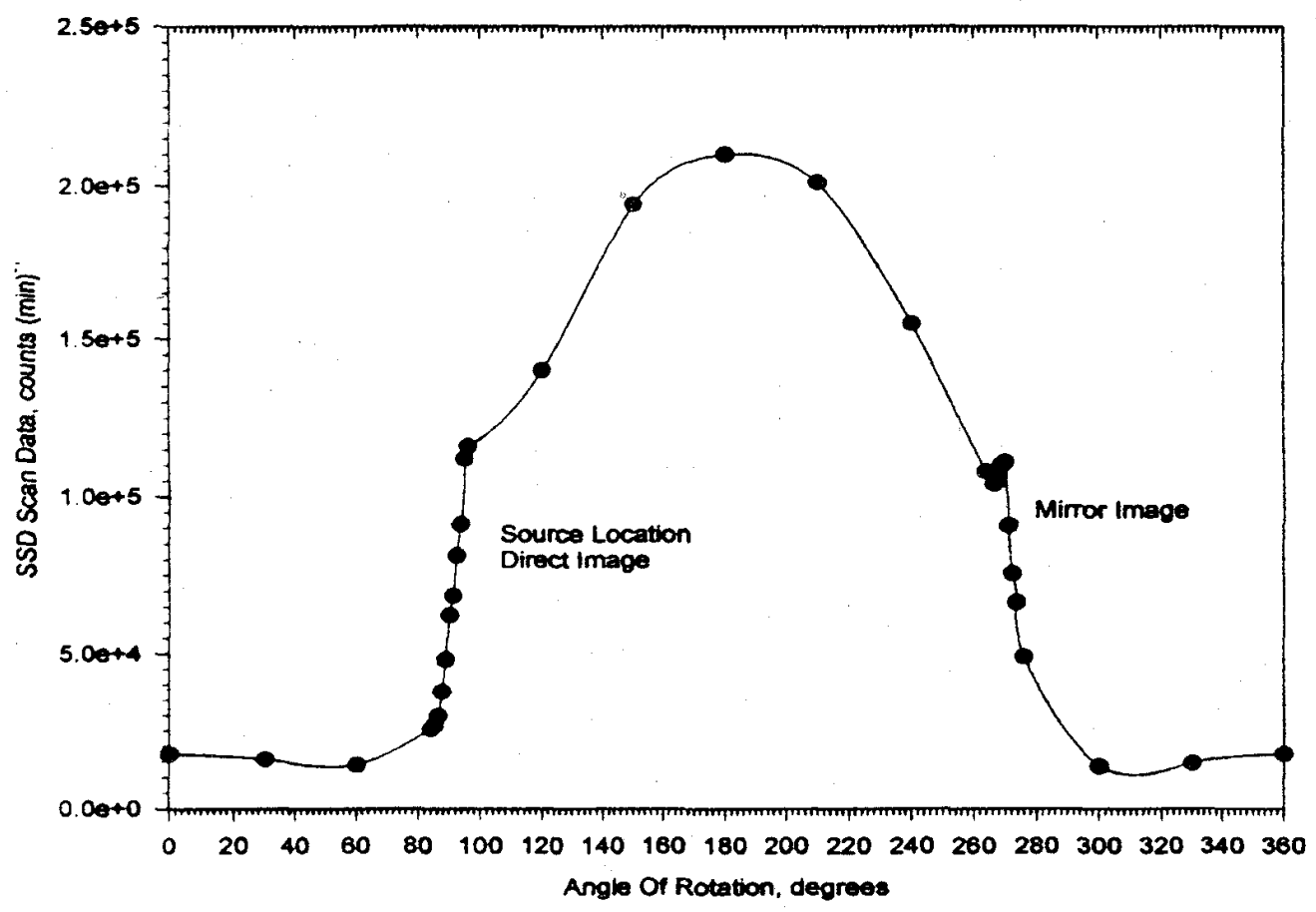

Fig. 4 Solid State Detector (SSD) scan of 174 keV X-ray photons.

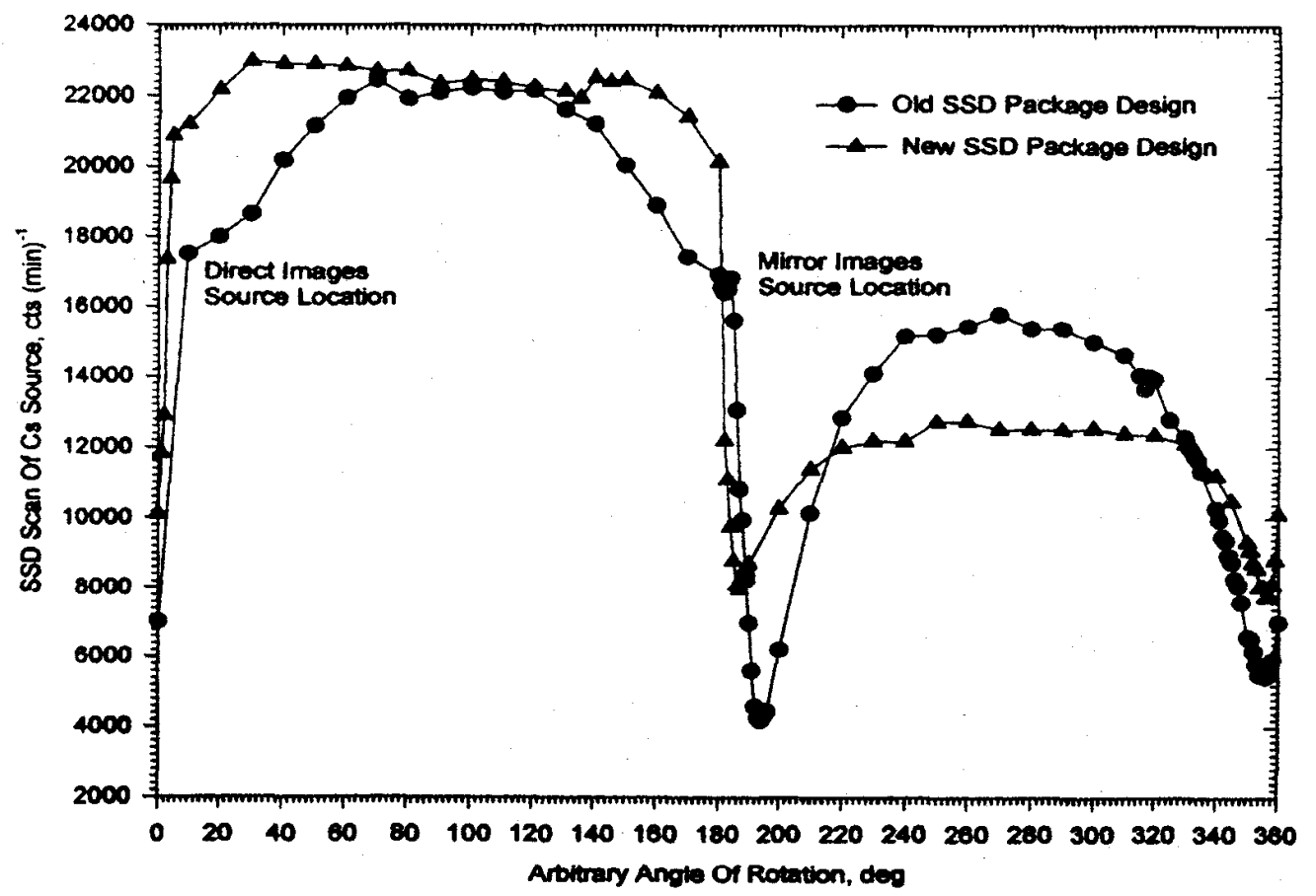

Fig. 5 Solid state Detector scane of ${ }^{137} \mathrm{Cs}$ point source for old and new peckage desions, 400 mrade $\mathrm{h}^{-1}$ at detector. 


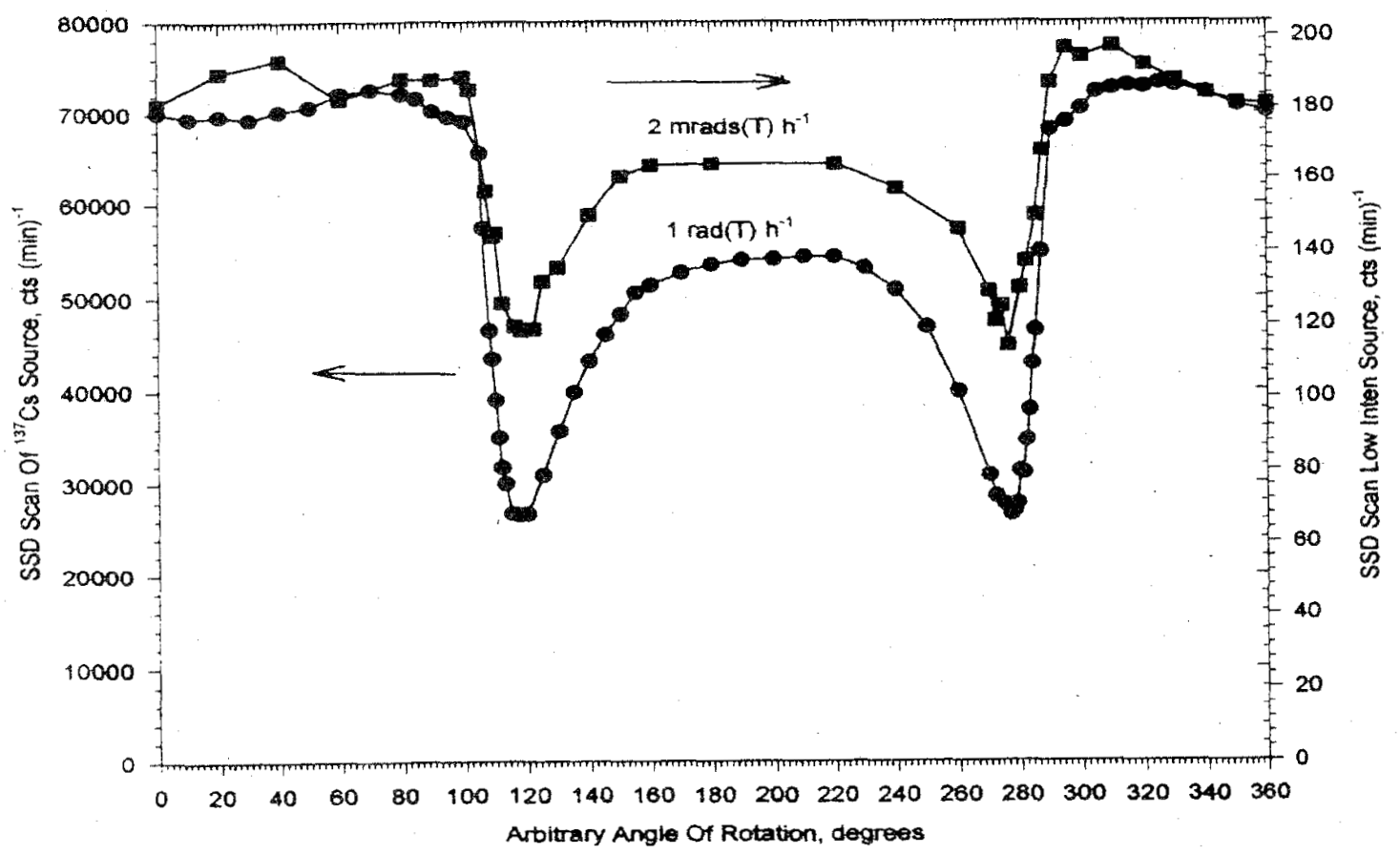

Fig. 8 Solid State Detector (SSD) scan ${ }^{137} \mathrm{Cs}$ point source, for two dose rates, 4 rad $(T) h^{-1}$ and $2 \mathrm{mrads}(\mathrm{T}) \mathrm{h}^{-1}$

higher dose rate is slightly better than for the lower one.

\section{Sumary of Results}

The sensitivity of the SSD sensor is about a factor of 1000 smaller than the SC device. A larger area diode would greatly increase the angular resolution and sensitivity of this type of directional sensor. The primary advantage of the SSD is its small size and ease of operation. Thus, it could be designed as a simpler field instrument than a scintilation sensor but it could not compete with the SC device for applications requiring high angular resolution. and sensitivity to radiation sources of low intensity.

\section{Conclusions}

It can be concluded from the results that a SSD sensor can be fabricated to scan point or large area sources of radiation to generate images of these sources. It was also demonstrated that the forwardscattered radiation from blocks of concrete when they were irradiated by ${ }^{60} \mathrm{Co}$ gamma rays can be scanned by a SC or SSD sensor to produce images of the targets, scatter patterns, and the primery source of unscattered radiation.

In principle, the angular resolution of the directional sensor is unlimited $(1,2)$. The limit is determined only by the accuracy of the instruments used to measure the angles. The limiting radiation source intensity is set by the sensitivity of the basic detector. In the case of this SSD type of sensor and the size of the diode used in this sensor, the angular resolution was a few degrees and the sensitivity was limited to a source intensity of about $1 \mathrm{mrad} / \mathrm{h}$. These limits could be improved by using a larger size diode or by stacking several diodes with low and high $z$ layers between them.

\section{References}

(1) S. Kronenberg, G. J. Brucker, E. Bechtel, and $F$. Gentner,

"Directional Detector for Arrays of Gamma Ray and X-ray Sources," Nucl. Instr. and Meth. in Phys. Res. A, $378,531-5401996$.

(2) S. Kronenberg, G. J. Brucker, E. Bechtel, F. Gentner, and $A$.

Iee, "Irocating and Imaging Sources of Gamma and X-radiation Directly or Through Thick Shields," Nucl. Instr. and Meth. In Phys. Res. A, 387, 4014091997.

(3) S. Kronenberg, and G.J. Brucker, "High-Angular Resolution Sensing of Gamma Rays in Space" " Proceedings of Society of Photo-optical Instrumentation Engineers, San Diego, published in Vol. 3116, July 271997. 